EPJ Web of Conferences 52, 06004 (2013)

DOI: $10.1051 /$ epjconf/20135206004

(C) Owned by the authors, published by EDP Sciences, 2013

\title{
Where do the highest energy CR's come from? and How does the Milky Way affect their arrival directions?
}

\author{
Philipp Kronberg ${ }^{1, a}$ \\ ${ }^{1}$ University of Toronto, Canada
}

\begin{abstract}
A "grand magnetic design" for the Milky Way disk clearly emerges within $\sim 1.5 \mathrm{kpc}$ of the Galactic mid-plane near the Sun [1], and reveals a pitch angle of $-5.5^{\circ}$ directed inward from the Solar tangential. This pitch angle can be expected to differ for Galactic disc locations other than ours. Above $\sim 1.5 \mathrm{kpc}$, the field geometry is completely different, and its 3-D structure is not yet completely specified. However it appears that the UHECR $\left(>5 \cdot 10^{19} \mathrm{eV}\right)$ propagation to us is not much affected by the halo field, at least for protons. I discuss new multi-parameter analyses of UHECR deflections which provide a conceptual "template" for future interpretations of energy-species-direction data from AUGER, HiRes etc., and their successors [2]. I show how the strength and structure of the cosmologically nearby intergalactic magnetic field, $\mathrm{B}_{I G M}$, is now well-estimated out to D $\sim 5$ Mpc from the Milky Way: - 20nG. These are the first VHECR data-based estimates of $\mathrm{B}_{I G M}$ on nearby supragalactic scales, and are also important for understanding and modeling CR propagation in the more distant Universe. CR Acceleration to the highest energies is probably a natural accompanying phenomenon of Supermassive Black Hole (SMBH)-associated jets and lobes [3]. I briefly describe what we know about magnetic configurations in these candidate sites for UHECR acceleration, and the first direct estimate of an extragalactic Poynting flux current, $\sim 3 \cdot 10^{18}$ Amperes [4, 5]. This number connects directly to SMBH accretion disk physics, and leads directly to ideas of how VHECR acceleration in jets and lobes, possibly involving magnetic reconnection, is likely to be common in the Universe. It remains to be fully understood.
\end{abstract}

\section{References}

[1] P. P. Kronberg and K. J. Newton-McGee, Remarkable Symmetries in the Milky Way Disc's Magnetic Field, Pub Astr. Soc. Australia, 28, 171-176, 2011

[2] H. Yüksel, T. Stanev, M.D. Kistler, P.P. Kronberg, The Centaurus A Ultrahigh-Energy CosmicRay Excess and the Local Extragalactic Magnetic Field Astrophysical Journal, 758, 16, 8 pp. 2012

[3] I.V. Pariev, S.A. Colgate, J.M. Finn, A Magnetic $\alpha-\omega$ Dynamo in AGN Disks. II. Magnetic Field Generation, Theories, and Simulations. Astrophys. J., 658, 1292007

[4] P.P. Kronberg, R.V.E. Lovelace, G. Lapenta, S.A. Colgate, Measurement of the Electric Current in a kpc-scale Jet, Astrophys J. Lett. 741, L15, 2011

[5] R.V.E. Lovelace, 1976, Dynamo model of double radio sources. Nature, 262, 649, 1976

\footnotetext{
appk101010@yahoo.com
}

This is an Open Access article distributed under the terms of the Creative Commons Attribution License 2.0, which permits unrestricted use, distribution, and reproduction in any medium, provided the original work is properly cited. 


\section{EPJ Web of Conferences}

\section{Slides}

The slides of the talk can be found on the website of the symposium ISVHECRI 2012: https://indico.desy.de/conferenceOtherViews.py?view=standard\& confId $=4594$ 